nephron

Experimental

Nephrology

and Genetics
Nephron 2016;132:227-237

DOI: $10.1159 / 000444267$
Received: February 19, 2015

Accepted after revision: January 15, 2016

Published online: February 26, 2016

\title{
The Critical Role of Innate Immunity in Kidney Transplantation
}

\author{
David Cucchiari Manuel Alfredo Podestà Claudio Ponticelli \\ Nephrology and Dialysis Unit, Humanitas Clinical and Research Center, Rozzano, Italy
}

\section{Key Words}

Dendritic cells · Graft rejection · Innate immunity .

Kidney transplantation

\begin{abstract}
For a long time now, kidney transplant rejection has been considered the consequence of either cellular or antibodymediated reaction as a part of adaptive immunity response. The role of innate immunity, on the other hand, had been unclear for many years and was thought to be only ancillary. There is now consistent evidence that innate immune response is a condition necessary to activate the machinery of rejection. In this setting, the communication between antigen-presenting cells and T lymphocytes is of major importance. Indeed, T cells are unable to cause rejection if innate immunity is not activated. This field is currently being explored and several experiments in animal models have proved that blocking innate immunity activation can promote tolerance of the graft instead of rejection. The aim of this review is to systematically describe all the steps of innate immunity response in kidney transplant rejection, from antigen recognition to T-cells activation, with a focus on clinical consequences and possible future perspectives.
\end{abstract}

(c) 2016 S. Karger AG, Basel (c) 2016 S. Karger AG, Basel

$1660-8151 / 16 / 1323-0227 \$ 39.50 / 0$

\section{Introduction}

The role of innate immunity in kidney transplantation has been overlooked for years, but now it is generally accepted that it represents the first step in rejection mechanisms and guides the development of adaptive immune response. Its role was elucidated by Janeway [1] in 1989, who postulated that non-self-recognition is initiated by a number of germ-line encoded receptors named pattern recognition receptors (PRRs). According to this model, PRRs recognize a repertoire of bacterial or viral molecules, called pathogen-associated molecular patterns (PAMPs), such as lipopolysaccharide and peptidoglycan. PAMPs recognition by PRRs leads to the production of several pro-inflammatory mediators that are essential in the first phase of inflammation and guides the subsequent development of adaptive immune response. However, this model could not explain the innate immunity activation in autoimmune diseases and allotransplantation, assuming that it is performed under sterile conditions. It was to the merit of Dr. Polly Matzinger in the nineties to clarify how innate immunity can also be activated by noninfectious stimuli. She expanded Janeway's hypothesis postulating that PRRs recognize as danger signals also self molecules that are released from dying or necrotic cells,

\section{KARGER}

E-Mail karger@karger.com

www.karger.com/nef
Dr. David Cucchiari

Nephrology and Dialysis Unit

Humanitas Clinical and Research Center

via Manzoni 56, IT-20089 Rozzano (Italy)

E-Mail david.cucchiari@gmail.com 
the so-called damage/danger-associated molecular patterns (DAMPs) [2]. According to this model, every injury to the graft leads to the production of DAMPs that potentially engage PRRs. Recently, Matzinger [3] noticed that Dr. Walter Land was the first to foresee the danger model before the publication of her theory [4], when he found out that a single shot of superoxide dismutase, a free radical scavenger, could reduce the long-term rate of rejection episodes in kidney transplant patients [5]. The aim of this review is to systematically describe all the steps of innate immunity response in kidney transplant rejection, from antigen recognition to T-cells activation. Finally, future perspectives based on experimental models will be discussed.

\section{Overview of Innate-Adaptive Immunity Interplay}

The communication between innate and adaptive immunity mainly relies on antigen presentation. The role of antigen-presenting cells (APCs) may be played by B cells, $\gamma \delta$ T-cells, macrophages and perhaps natural killer (NK) cell [6]. However, in most cases, the cells presenting the antigen to immune competent lymphocytes are dendritic cells (DCs), which provide stronger activation signals than other cells. Before presenting the antigen, DCs need to change from immature cells specialized for antigen capture into mature cells that activate $\mathrm{T}$ lymphocytes. This phenotypic transition relies on the presence of an inflammatory micro-environment and represents an essential prerequisite for $\mathrm{T}$ cells activation. The quiescent DCs, indeed, release chemokines and cytokines that favor Treg differentiation [7]. When DAMPs and/or PAMPs are present instead, mature DCs can efficiently activate naïve T cells. This happens in lymph nodes, where T cells are attracted by DCs-released chemokines. Naïve T cells require 3 signals to be activated and avoid death and/or tolerance. The first signal is provided by the contact between the alloantigen presented by APCs and the specific receptor located on the surface of T cells. However, this is an anergic/apoptotic signal. A second signal (costimulation) is needed to rescue $\mathrm{T}$ cells from apoptosis and to activate them. This is given by the contact between proteins located on the APCs, such as CD80 and CD86 of the B7 family, and CD28 on the surface of T cells. Also important is the contact between $\mathrm{CD} 40$ and its ligand $\mathrm{CD} 40 \mathrm{~L}$ also called CD 154. On the other side, some other receptors on T cells, such as cytotoxic T lymphocyte antigen 4 (CTLA-4) and programmed death 1, engage B7 proteins and inhibit proliferation. CTLA-4 has a higher affinity for
B7 receptors on APCs, up to 100 times higher than CD28. When APCs have not been activated by DAMPs or PAMPs, they express low levels of CD80 and CD86. CTLA-4, due to its higher affinity, prevents CD28 engagement by the B7 family and, thus, impairs T-cell activation [8]. On the other side, when APCs are activated by noxious stimuli, B7 receptors increase on DCs and become available for CD28 engagement and T-cell activation. This is a good example of how the inflammatory milieu (i.e. the balance between stimulatory and inhibitory costimulatory molecules) can decide the T-cell destiny (fig. 1). Furthermore, a third signal is also needed to guide T-helper cells differentiation [9]. This is given by some cytokines, such as interleukin (IL)-12, IL-23 and TGF-beta, which regulate the differentiation of Th subsets [10].

\section{Who Recognized the Danger? The Role of PRRs}

Therefore, the initiation of allo-response in renal transplantation needs DAMPs recognition by PRRs of the innate immunity. This enables APCs maturation and subsequent activation of the adaptive immune response, as discussed previously. But how can the danger be perceived? Innate immunity can rely on a wide array of cellular and soluble receptors to carry out this task.

\section{Cellular Receptors}

The most studied and better characterized cellular receptors are toll-like receptors (TLRs), NOD-like receptors (NLRs) and RIG-I-like receptors, while soluble mediators include complement and pentraxins. TLRs are integral membrane glycoproteins located either on the external surface of the cell membrane or on the endosomes and are expressed on DCs, macrophages, B cells, NK cells, endothelial cells and many other cells [11]. To date, human functional TLRs are divided into 2 subgroups, depending on their localization: TLR1, TLR2, TLR4, TLR5, TLR6 and TLR11 are expressed on cell surfaces and recognize mainly microbial membrane components, while TLR3, TLR7, TLR8 and TLR9 are expressed only in intracellular vesicles and recognize microbial nucleic acids. Different intracellular pathways of signal transduction have been described for these 2 groups of receptors. TLRs expressed on cells surface, upon antigen binding, dimerize and transduce the inflammatory signal through the intracellular toll/IL-1 receptor (TIR) domain. TIR dimerization on the cytoplas- 


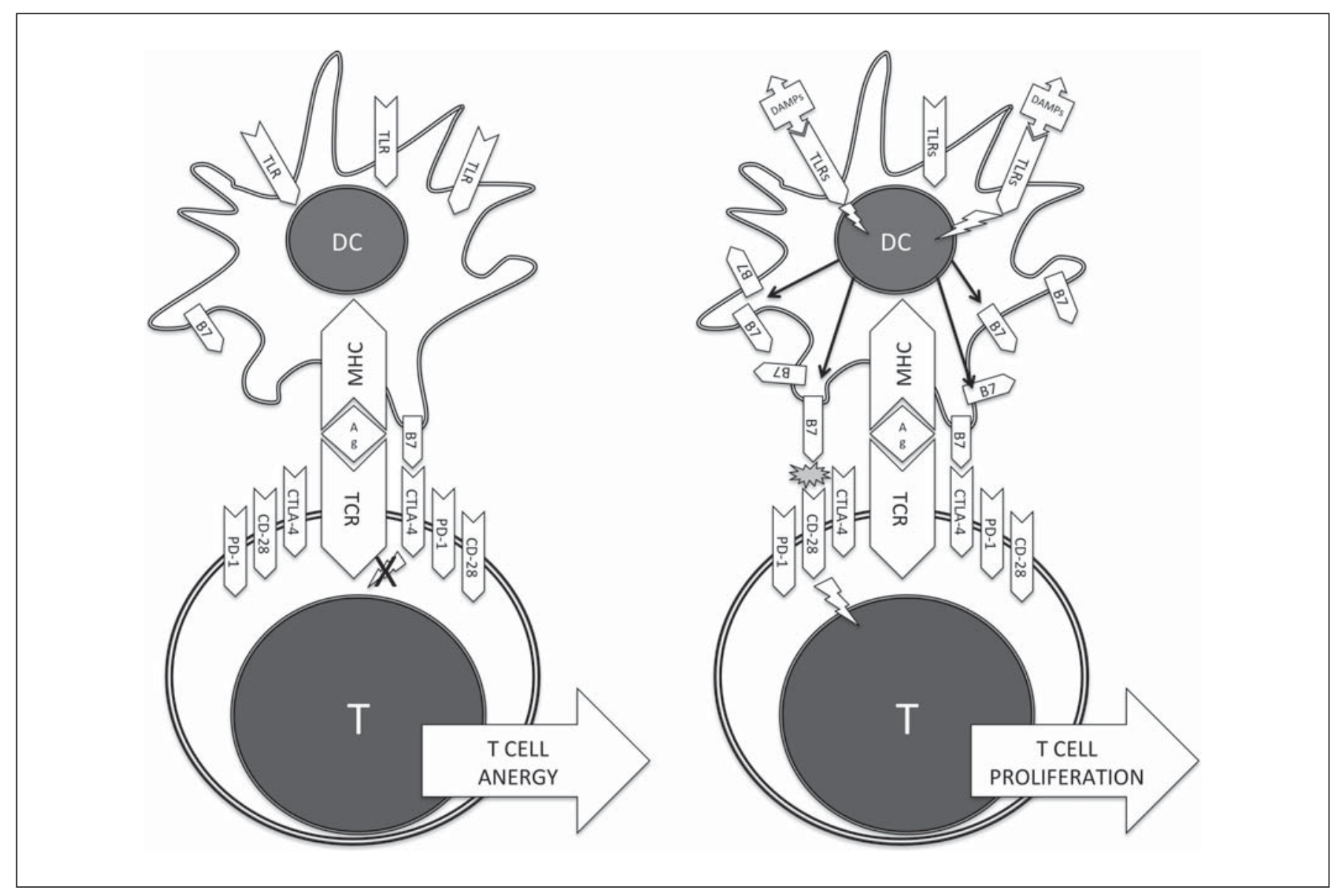

Fig. 1. When DCs have not been activated by noxious stimuli (left), they express low levels of B7 proteins (such as CD80, CD86). Some receptors on T cells, such as CTLA-4, prevent CD28 engagement by the proteins of B7 family, due to their higher affinity. This avoids inappropriate $\mathrm{T}$-cell activation and proliferation when an inflammatory medium is absent. On the other side (right), when
DAMPs engage PRRs such as TLRs, the expression of CD80 and CD86 (B7) increases notably on the surface of DCs. This allows the engagement of CD28 by B7 proteins, thus providing the necessary co-stimulation that $\mathrm{T}$ cells need to proliferate upon antigen recognition. For a more detailed description, see the text. TCR $=\mathrm{T}$ cell receptor; $\mathrm{PD}-1=$ programmed death $1 ; \mathrm{Ag}=$ antigen; $\mathrm{T}=\mathrm{T}$ cell . mic side of plasma membrane recruits adapter proteins, such as myeloid differentiation primary response gene 88 (MyD88) and MyD88-adapter, in turn activate intracellular kinases responsible for signal transduction, including IL-1 receptor associated kinases (IRAK) [12], I $\kappa B$ kinases, and protein kinase $C$ [13]. This results in the translocation of transcription factors into the nucleus, such as AP-1 and nuclear factor kappa-light-chain-enhancer of activated B cells (NF- $\kappa$ B) [14], where they activate the transcription of pro-inflammatory genes, including cytokines, chemokines, adhesion and costimulatory molecules (fig. 2).

On the other hand, TLRs located on the intracellular vesicles, when engaged by viral moieties, initiate a signaling cascade that leads to the transcription of type I inter- ferons through the translocation of the interferon response factors (IRFs) into the nucleus, such as IRF3 (fig. 2).

NLRs are intracellular sensors that collaborate with TLRs in recognizing PAMPs and DAMPs in order to activate NF- $\kappa B$ and MAP kinases [15]. These receptors are expressed in the epithelial tubular cells and seemed to be crucially involved in the inflammatory response triggered by IRI in a murine model [16]. NOD leucine-rich repeat and pyrin domain containing protein (NLRPs), including NLRP3, are a distinct subfamily of NLRs that, following activation by PAMPs or DAMPs, oligomerize and recruit both the adaptor protein ASC and the inactive form of protease caspase- 1 to form a multimolecular complex termed inflammasome that has proteolytic properties 


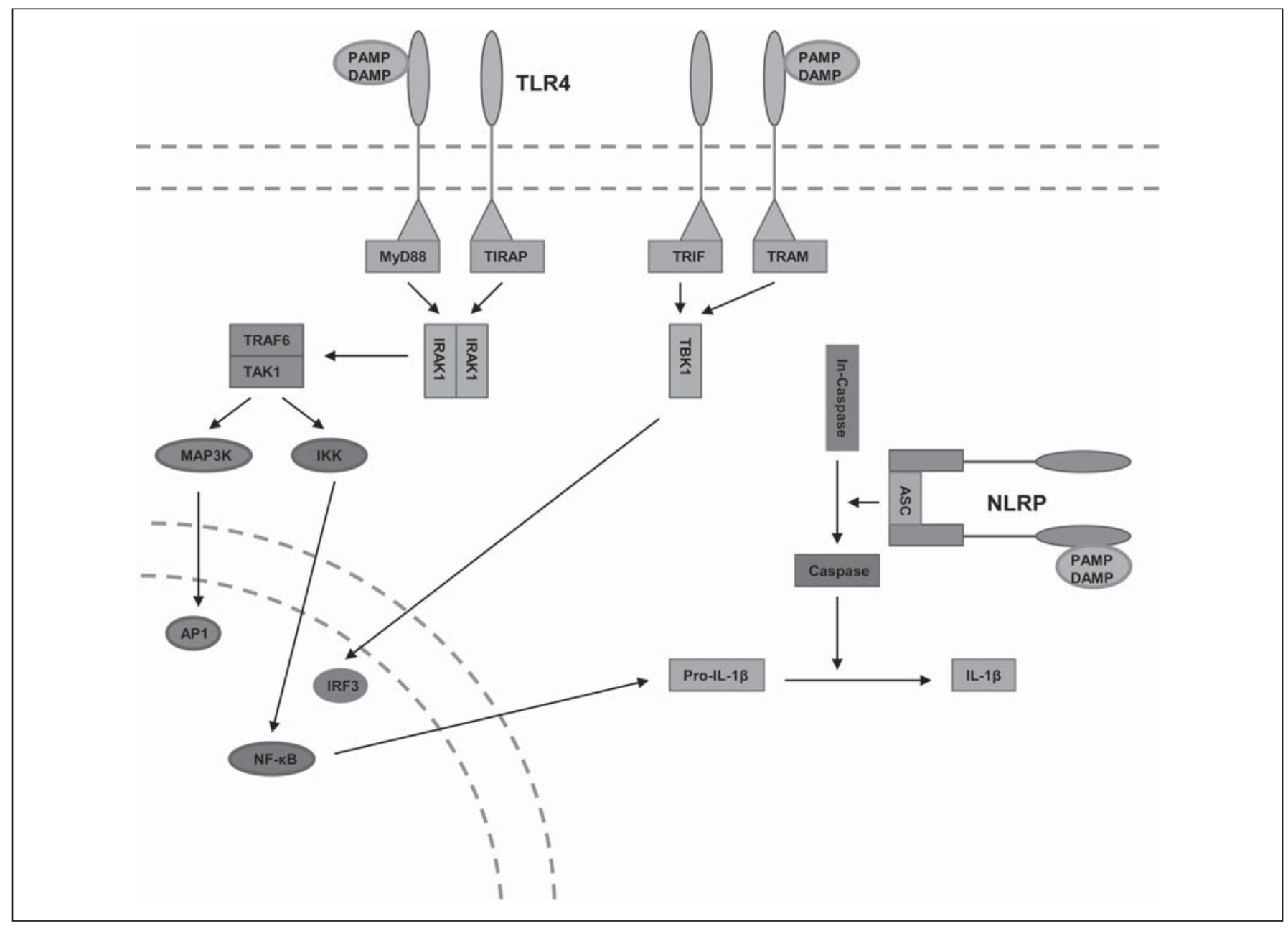

Fig. 2. TLRs engagement with either PAMPs or DAMPs activates an intracellular cascade of kinases that leads to the production of transcription factors, such as AP1, NF- $\kappa$ B and IRF3, which regulate the transcription of many inflammatory genes. TLRs expressed on the cell surface, upon antigen binding, dimerize and transduce the inflammatory signal through the intracellular TIR domain. TIR dimerization on the cytoplasmic side of plasma membrane recruits adapter proteins, such as MyD88 (also known as TIRAP) and MyD88-adapter like (Mal), which in turn activate intracellular kinases responsible for signal transduction, including IRAK,

and is essential to activate by cleavage some proinflammatory cytokines, such as IL-1 $\beta$ and IL-18 (fig. 2) [17].

Finally, RIG-I-like receptors are intracellular receptors that intercept and recognize the intracellular viral replication through the direct interaction with the dsRNA produced by viral RNA to form the genome [18].

For readers who are particularly interested to know more on this subject, some excellent reviews have been recently published about the role of PRRs in general [19] and, more specifically, in kidney diseases [20].
MAP-kinases, IKKs, and protein kinase C. NLRP3 activation following DAMPs sensing builds up a multimolecular complex called inflammasome that, through caspase-1, cleaves and activates some pro-inflammatory cytokines, such as IL-1 and IL-18, into the active form. AP1 = Activation protein-1; IKK = I $\kappa$ B kinase; In-caspase $=$ inactive caspase; $\mathrm{MAP} 3 \mathrm{~K}=\mathrm{MAP} 3$ kinase; $\mathrm{TAK} 1=\mathrm{TGF} \beta$ activated kinase $1 ;$ TBK $=$ TANK binding kinase; TIRAP $=$ TIR domain containing adaptor protein; TRAM = TRIF-related adaptor molecule; TRAF6 $=$ TNF receptor-associated factor 6 ; TRIF $=$ TIR-domain-containing adapter-inducing interferon- $\beta$.

\section{Soluble Receptors}

Besides the cellular arm, the innate immunity also comprises humoral components, namely complement and pentraxins.

The complement system includes more than 30 proteins both in plasma and on cell surfaces and presents 3 distinct activation cascades, the classical, alternative and mannose-binding lectin pathway [21]. Although the complement system plays important protective roles against infection and tumors, it can be harmful to the 
graft through activation of both inflammatory response and coagulation cascade [22]. A number of events including brain death of the donor, IRI, infection, coagulation, and any type of tissue damage can trigger systemic and renal activation of complement in this setting. Curiously, the lectin pathway seems to be the most commonly involved during IRI. This happens because the mannosebinding lectin can bind some oligosaccharides other than mannose that are expressed by necrotic and late apoptotic cells [23]. Locally produced C5a and C3a are critical for the activation of adaptive immunity, since they enable the necessary costimulation that $\mathrm{T}$ cells need to proliferate upon engagement with APCs.

Pentraxins are a family of multimeric pattern-recognition proteins that can be divided into short pentraxins and long pentraxins [24]. Short pentraxins, that is, C-reactive protein and serum amyloid $\mathrm{P}$-component, are opsonins produced in the liver in response to proinflammatory cytokines, such as IL- 6 and contribute to innate immunity responses. The prototypic long pentraxin is pentraxin 3 (PTX3), a fluid phase pattern recognition molecule that is produced by DCs and macrophages in response to TLRs and inflammatory cytokines. PTX3 has antibody-like properties and binds $\mathrm{Clq}$ activating the complement cascade [25]. Moreover, it is expressed by renal proximal tubular cells [26]. Its role in renal transplantation remains elusive at the moment: whether it exerts beneficial or harmful effects is still unclear $[27,28]$.

\section{What Is Recognized? The Essential Role of DAMPs}

DAMPs include all the substances that are released from damaged tissues and that can be recognized by the specific receptors of innate immunity (PRRs). A large number of DAMPs released by damaged or necrotic cells has been identified so far and several functional experiments in animal models have confirmed their essential role in the development of rejection. DAMPs can be broadly classified into those that are actively expressed or secreted by damaged cells and those that are passively released by dying cells. DCs, lymphocytes, inflammasomes and IgM antibodies can recognize them. Moreover, damaged extracellular proteins, such as fragments of hyaluronan, heparin sulphate, fibronectin and biglycan, can act as efficient DAMPs [29]. One of the most studied proteins is the high-mobility group box 1 (HMGB1) protein, whose role is to regulate chromosomal stability. It is shed from dying or damaged cells in the extracellular matrix, where it has chemotactic effects for virtually all immune

Innate Immunity in Kidney

Transplantation cells and activates APCs through engagement with TLR2, TLR4 and the receptor for advanced glycation end (RAGE) products [30]. Heat shock proteins (HSPs) are a family of intracellular chaperones involved in folding and unfolding of newly synthetized polypeptides. In stress conditions, such as hypoxia, their production and secretion are enhanced and leads to APCs' activation through TLRs engagement [31]. HSP70 expression is already increased in kidneys removed from deceased donors; this precedes a more dramatic upregulation when reperfusion later occurs [32]. Leakage of ATP (extracellular ATP) and uric acid out of damaged cells can activate the inflammasome, leading to the production of IL- $1 \beta$ and IL-18. In particular, extracellular ATP induces P2X8-dependent pores formation on cell membranes by the assembly of pannexin-1 hemichannels. This allows the entry of extracellular ATP itself and other extracellular DAMPs into the cells, where they can engage NLRP3 in order to activate the inflammasome [29]. Coagulation proteases, besides generating fibrin, are involved in the inflammation process through engagement of the so-called proteaseactivated receptors on endothelial cells, DCs and T cells. These receptors are activated upon cleavage by the coagulation proteases and increase the expression of adhesion molecules and promote the alloantigen-primed T-cell recruitment [33].

\section{Innate Immunity Activation in Kidney Allotransplantation}

A number of factors may activate innate immunity in allotransplantation. In the brain-deceased donor, the intracranial hypertension triggers a massive release of cytokines and growth factors with consequent activation of cell-adhesion molecules and leukocyte infiltration in the kidney [34]. Another consequence of brain death is local upregulation and the activation of complement that can contribute to create an inflammatory environment [35]. The manipulation of the kidney during removal may also produce mechanical injury. However, the most dangerous and best studied mechanism of graft damage is represented by IRI. Ischemia leads to poor ATP generation and intracellular acidosis through the activation of the glycolytic pathway. This deranges the activity of sodium/ potassium ATPase pumps and disrupts the osmotic equilibrium. As a result, cells swell due to the osmotic stress and start to express adhesion molecules, such as P-selectin, E-selectin and ICAM-1, that will promote leukocytes infiltration of the graft in the reperfusion phase. The re- 
perfusion phase leads to the production of a huge quantity of reactive oxygen species (ROS). In the first minutes after reperfusion, ROS production is thought to originate from endothelial cells that have been damaged by the previous phase of ischemia. The mitochondrial electron transport chain is the system mostly involved in ROS production. Leakage of electrons from damaged mitochondria generates superoxide anions, hydrogen peroxide, hydroxyl radicals, hypochlorous acid and singlet oxygen. Afterwards, a second burst of ROS production is due to neutrophils migration to the graft, where they contribute to tissue damage and promote the subsequent recruitment of alloreactive T cells [36].

Oxidative and genotoxic stress determine cellular damage that often result in regulated forms of necrosis, which may involve mediators such as serine/threonine protein kinase RIP1-RIP3 (necroptosis) or glutathione peroxidise 4 (ferroptosis) $[37,38]$. These types of necrosis may potentially lead to massive release of intracellular DAMPS.

It is worthy to consider that inflammation does not only drive tissue injury but also favors its repair. TLR2 activation by DAMPs engagement determines clonal expansion and activation of renal progenitor cells located along the thick limbs of the renal tubule, a process that leads to hastened regeneration. In addition, following TLR4 activation, the release of IL- 22 by macrophages and DCs activates a specific receptor on these tubular cells that favors the reparative process. Selective blockade of TLR signalling in the recovery phase of IRI leads to a consistent delay in epithelial regeneration $[39,40]$. Failure to resolve acute inflammation following injury can lead to the development of chronic inflammation that contributes to the pathogenesis of graft dysfunction [41]. DAMPs themselves may indeed affect renal fibrosis, an aberrant process, which tends to re-establish tissue homeostasis through deposition of extracellular matrix. The DAMPsmediated activation of fibroblasts, pericytes and mesangial cells leads to fibrosis via the induction of molecular pathways that result in TGF- $\beta$ signalling and epithelialmesenchymal transition of these cells [42].

Timely regulation of inflammation and recovery, both active processes, is subjected to a wide array of factors, in which innate immunity mediators play a consistent role. Recently, increasing attention has been paid to the influence of macrophage phenotype in the different phases of ischemia-reperfusion injury. Classically activated M1 macrophages are considered proinflammatory cells, which enter the site of injury along neutrophils following the release of chemokines (such as CX3CL1, released by renal endothelial cells). Neutrophils apoptosis and their subsequent phagocytosis switch macrophages towards an anti-inflammatory phenotype (M2, alternatively activated) that contributes to inflammation resolution and promotes healing [43]. A consistent number of mediators are implied in this phenotypic switch: genetic or pharmacological inhibition of macrophage colony stimulating factor 1 (CSF-1), which is released by damaged renal epithelial cells, resulted in a reduction of M2 polarization and impaired recovery [44]. Of note, CSF-1 also acts on renal tubular cells themselves via a specific receptor, which is upregulated upon injury. This signalling results in decreased apoptosis, increased proliferation and lessened interstitial fibrosis [45]. Similarly, genetic depletion of IRAK-M, a macrophage-specific inhibitor of TLR and IL$1 \beta$ signalling induced after their activation, resulted in interstitial accumulation of M1 macrophages with subsequent interstitial fibrosis and tubular atrophy [46].

If IRI represents the major early event that triggers innate immunity, it should be pointed out that every injury to the graft - including infection, drug toxicity, tissue damage - may theoretically lead to the production of PAMPs or DAMPs with consequent activation of the innate immunity. Of particular importance, pathogen- or allograft-derived antigens can activate innate immunity response and create a pro-inflammatory milieu. Some T cells specific for microbial peptides presented by self-major histocompatibility complex (MHC) molecules can cross-react with allogeneic MHC, while bacterial superantigens can directly activate large populations of T cells. Therefore, infections experienced before transplantation can give rise to memory alloreactive $\mathrm{T}$ cells that may be more resistant to immunosuppression than naïve $\mathrm{T}$ cells [47]. Infections occurring late after transplantation may, in theory, elicit pro-inflammatory signals that activate tolerant $\mathrm{T}$ cells by enabling their escape from immunosuppression and/or peripheral mechanisms of tolerance, thereby precipitating rejection [48]. Activation of innate immunity by trivial triggers could explain the occurrence of some cases of unexpected late deterioration of renal function in patients with stable transplants. Depending on the balance between effector and regulatory $\mathrm{T}$ cells, these events may result in innocent renal function fluctuations or progression to irreversible renal failure. Recent emphasis has been given to the complex interplay between gut microbiota and innate and adaptive immunity. For instance, in an animal model of IRI-induced AKI, germ-free mice had worse structural and functional outcomes compared to mice that were colonized with wild-type gut flora. Authors suggested that a more pro- 
nounced T helper 1 phenotype in germ-free mice could have promoted a worse injury [49].

It is known that fecal microbiota undergoes dramatic changes after kidney transplantation, resulting in the overgrowth of some bacterial species and overall reduction in microbial diversity. The resulting changes in gut microbiota have been associated with the development of post-transplant diarrhoea and Enterococcus-related infectious complications. Moreover, patients with acute rejection have substantial different gut microbial composition [50]. Whether microbiota has an active role in acute rejection mechanisms is a fascinating hypothesis that deserves further validation. Indeed, changes in gut microbiota may represent only the consequence of immunosuppression and antibiotic prophylaxis given to transplant patients [51]. However, its role cannot be undervalued since drug metabolism can be affected by changes in gut flora, as Lee et al. [52] recently demonstrated that Faecalibacterium prausnitzii abundance in the first week after transplantation had an impact on tacrolimus dose requirements.

\section{Antigen Presentation and Activation of Adaptive Immunity}

After antigen uptake, phenotypic transition and migration to lymphoid tissues, DCs can finally present the antigen to immunocompetent cells. In kidney transplantation, antigen presentation to T cells has 3 different modalities: (i) direct presentation: the donor's DCs present directly the antigen to $\mathrm{T}$ cells (ii); indirect presentation: recipient's DCs intercept the antigen, become mature, and present the antigen to T cells; (iii) semi-direct presentation: recipient's DCs take up membrane fragments of donor's DCs membranes for presentation through indirect pathway [53]. Once T lymphocyte has been activated, there is a large influx of $\mathrm{Ca}^{2+}$ ions into the cytoplasm leading to the activation of calcineurin, a phosphatase that dephosphorylates a family of proteins called nuclear-factor of activated T cells (NFAT). After dephosphorylation, NFAT can enter the nucleus and stimulates the synthesis of IL-2, a potent pro-proliferative cytokine that expands the $\mathrm{T}$ clone itself and strengthens the adaptive response against the allo-antigen presented. The contact with APCs also activates the mitogen-activated protein kinase and the protein kinase $\mathrm{C}-\mathrm{NF}-\kappa \mathrm{B}$ pathways. This results in the activation of IL-2, IL-2 receptor, NF- $\kappa B$, and a further activation of APCs [54]. The ligand of IL-2 to its receptor (CD25), together with IL-15, activates a kinase, called Ja- nus kinase 3, and delivers growth signals through the family of phosphoinositide-3 kinase (PI3-K) which, together with a protein-kinase $\mathrm{B}$ (AKT), activates a cascade of other kinases that provide the signals for cell proliferation [55]. The downstream effector of PI3-K is the mammalian target of rapamycin (mTOR), which regulates cell growth and proliferation (fig. 2, 3). mTOR phosphorylates the $40 \mathrm{~S}$ ribosomal 6 kinase and cyclin-dependent kinases, providing the transduction of proliferative signals to $\mathrm{T}$ cells [56]. After receiving all these signals, activated $\mathrm{T}$ lymphocytes require the synthesis of nucleotides to proliferate. Under the regulation of IL-12 released from macrophages, CD4+ $\mathrm{T}$ lymphocytes differentiate into Th1 and Th17 subsets [57]. Furthermore, CD4+ cells can also activate $B$ cells and induce their differentiation into memory and plasma cells [58].

\section{Future Approaches}

In addition to operative protection of the graft, several of the innate immunity pathways described above may theoretically be exploited to induce tolerance instead of rejection. Possible ways to inhibit innate immunity activation include PRRs and DAMPs antagonism and costimulation blockade.

Among PRRs, TLRs and their adapter molecules, such as MyD88 and TIR-domain-containing adapter-inducing interferon- $\beta$, are the best studied so far. TLR2 and TLR4 are upregulated following ischemia or nephrotoxic drugs playing a pro-inflammatory role [59] that may be prevented in mice with genetic deficiency of these receptors or treated with TLR2-antisense [60]. MyD88 deficiency is associated with an altered balance of Tregs over Th17 cells that promotes tolerance [61], although graft rejection can occur independently of MyD88 [62]. Even in humans, the importance of the TLRs machinery in generating alloimmune response has been highlighted. Krüger et al. [63] demonstrated that donor kidneys bearing a nonsense mutation of TLR-4 had an increased rate of immediate graft function along with a reduced expression of some inflammatory mediators at renal biopsy, such as TNF-alpha and MCP-1, whose messenger RNA (mRNA) was found to be significantly reduced compared to patients bearing the wild-type gene. Following these observations, increasing attention has been directed towards the development of compounds able to modulate TLR signalling. For instance, OPN-305, the first humanized IgG4 monoclonal antibody against TLR2 has been tested in a phase I randomized, double-blind, placebo- 


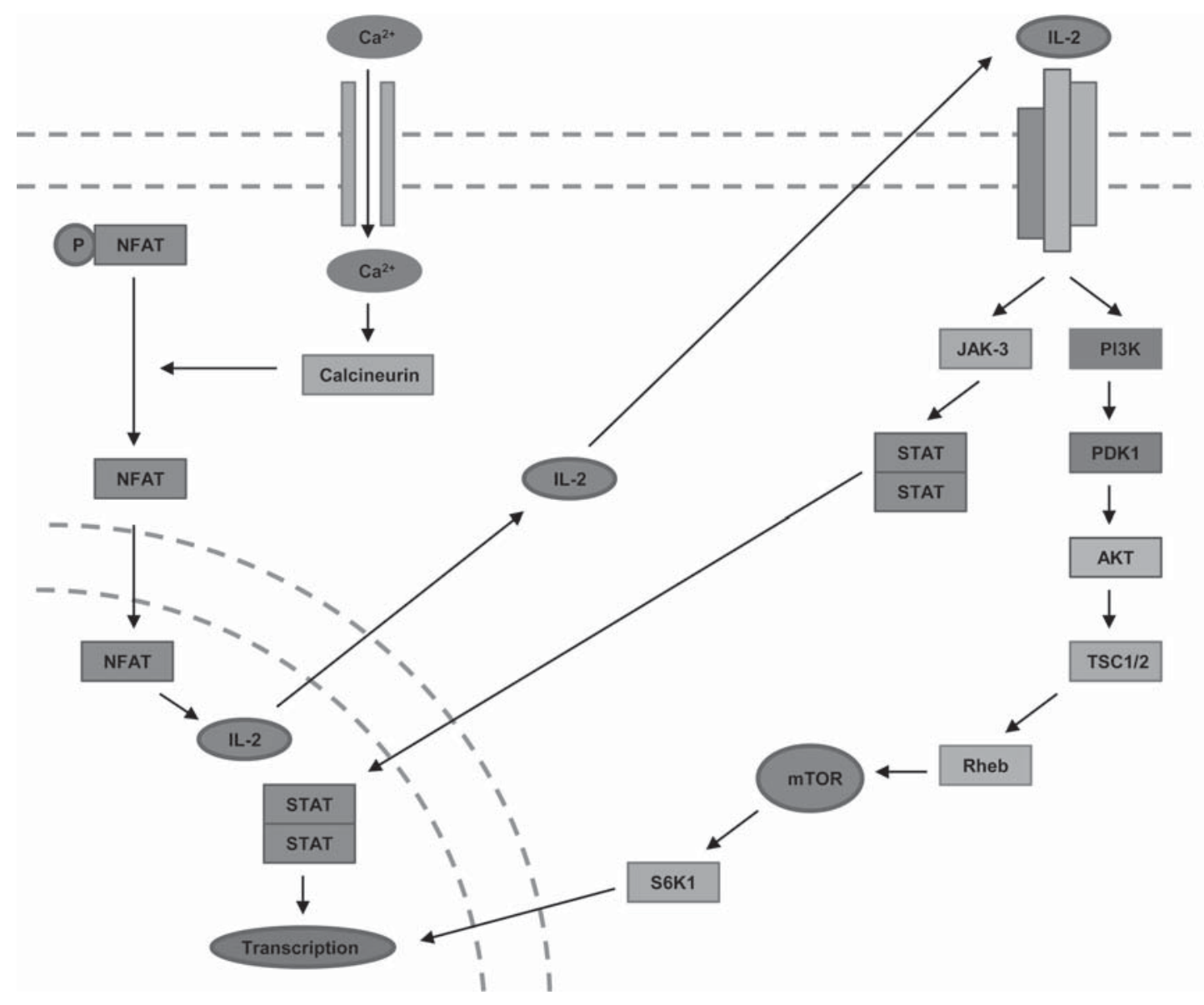

Fig. 3. T cells that are activated by an efficient antigen presentation by DCs increase their cytoplasmic calcium concentration through the opening of calcium channels. The increase in cytoplasmic $\mathrm{Ca}^{2+}$ activates calcineurin, a phosphatase that dephosphorylates NFAT. This allows the entry of NFAT into the nucleus, in which it activates the transcription of IL-2, a potent pro-proliferative cytokine. The pro-proliferative signal of IL- 2 is further mediated by the JAK/
STAT pathway and by the PI3-K pathway, with the latter that activates mTOR, an essential kinase that regulates cell growth and proliferation. JAK3 = Janus kinase 3; PDK1 = phosphoinositidedependent kinase-1; Rheb = Ras homolog enriched in brain; STAT = signal transducer and activator of transcription; TSC1/2 = tuberous sclerosis $1 / 2$.

Tir8(-/-) mice have increased TNF-alpha and IL-6 secretion from DCs and worse renal graft outcome [66].

Regarding DAMPs, several strategies have been based on HMGB-1 inhibition using molecules that compete with its receptors, such as TLR4 and RAGE. The discovery of a TLR4 cysteine residue critical for HMGB1 binding may favor selective inhibition of this DAMP [67]. In murine models, mice treated with anti-HMGB1 antibodies at the time of transplantation have less tubulointerstitial infiltration by neutrophils and macrophages and reduced apoptosis of tubular epithelial cells [68]. As an alternative, encouraging results have been obtained with direct HMGB1-targeting, leading to the identification of 
compounds that directly bind HMGB1, ranging from small natural or synthetic molecules, such as glycyrrhizin and gabexate mesilate, to HMGB1-specific antibodies, peptides, proteins as well as bent DNA-based duplexes [69].

Beyond the inhibition of danger recognition (i.e. the PRRs-DAMPs interplay), T-cell activation by DCs may be altered in order to induce tolerance. This may be accomplished by blocking costimulation, the signal that rescues $\mathrm{T}$ cells from apoptosis and leads to clonal expansion. In this field, one of the best studied molecules is CTLA-4, which prevents CD80-CD86 engagement to CD28 due to its higher affinity to the ligand. Several strategies proved to be efficient in preventing kidney transplant rejection in mouse and non-human primates models, from the use of recombinant CTLA4-Ig to the employment of monoclonal antibodies directed against CD40, CD28 and CD86 [70].

Further attempts to prevent innate immunity activation by IRI may be based on the inhibition of caspase-1, inflammasome, complement and NF- $\mathrm{BB}$. In 2006, Gilmore and Herscovitch [71] counted 785 inhibitors of NF- $\mathrm{kB}$ including anti-oxidant products, proteasomes and proteasome-inhibitors, I- $\kappa \mathrm{B}$ phosphorylation and degradation inhibitors, and other aspecific ones. Some of these inhibitors have been used in oncology and inflammatory diseases, but compounds designed as specific NF- $\kappa \mathrm{B}$ inhibitors are not yet clinically available for kidney transplantation. Other possible targets may be represented by micro-RNAs, a class of small endogenous non-coding RNAs that regulate gene expression through the translational control of mRNAs. They have recently emerged as key regulators of TLR-signalling pathways, and new technologies for the identification and manipulation of miRNA expression in vivo are now available [72]. Of course, the knowledge of innate immunity pathways remains incompletely understood and every new discovery in the field seems to challenge the old beliefs. For example, it has been recently demonstrated that innate sensing of allogeneic non-self can occur without the presence of DAMPs through monocytes-derived DCs [73]. Moreover, it should be highlighted that the clinical scenario is really different from clean facilities and sterile laboratories: humans are exposed to an assortment of pathogens and other environment perturbations that nullify most of the attempts to selectively block a specific signalling pathway. Therefore, the redundancy of the system may justify why the success observed in targeting innate immunity pathways in animal models has not been confirmed in humans yet.

\section{Authors' Contributions}

D.C. and M.A.P. contributed equally to the writing of all parts of the manuscript, C.P. conceived the idea of the manuscript, contributed to the writing of the manuscript and critically reviewed the final draft.

\section{Disclosure Statement}

C.P. has been consultant of Novartis, Italy until December 2011. In the last 2 years, he received honoraria for invited lectures from Novartis, Cilag-Janssen, Astellas, University of Calgary (Canada), North Shore Hospital of New York (USA), University of Zurich $(\mathrm{CH})$.

\section{Statement of Ethics}

This study did not require informed consent nor review/approval by the appropriate ethics committee.

\section{References}

1 Janeway CA Jr: Approaching the asymptote? Evolution and revolution in immunology. Cold Spring Harb Symp Quant Biol 1989;(54 pt 1):1-13.

2 Matzinger P: The danger model: a renewed sense of self. Science 2002;296:301-305.

3 Matzinger P: The evolution of the danger theory. Interview by Lauren Constable, Commissioning Editor. Expert Rev Clin Immunol 2012;8:311-317.

4 Matzinger P: Tolerance, danger, and the extended family. Annu Rev Immunol 1994;12: 991-1045.

5 Land W, Schneeberger H, Schleibner S, et al: The beneficial effect of human recombinant superoxide dismutase on acute and chronic rejection events in recipients of cadaveric renal transplants. Transplantation 1994;57: 211-217.

-6 Vivier E, Raulet DH, Moretta A, Caligiuri MA, Zitvogel L, Lanier LL, et al: Innate or adaptive immunity? The example of natural killer cells. Science 2011;331:44-49.

7 Mahnke K, Schmitt E, Bonifaz L, Enk AH, Jonuleit $\mathrm{H}$ : Immature, but not inactive: the tolerogenic function of immature dendritic cells. Immunol Cell Biol 2002;80:477483.

8 Hubo M, Trinschek B, Kryczanowsky F, et al: Costimulatory molecules on immunogenic versus tolerogenic human dendritic cells. Front Immunol 2013;4:82.

-9 Curtsinger JM, Mescher MF: Inflammatory cytokines as a third signal for T cell activation. Curr Opin Immunol 2010;22:333-340.

10 Schmitt N, Ueno H: Regulation of human helper $\mathrm{T}$ cell subset differentiation by cytokines. Curr Opin Immunol 2015;34:130-136.

11 Imler JL, Hoffmann JA: Toll and toll-like proteins: an ancient family of receptors signaling infection. Rev Immunogenet 2000;2:294-304.

$\checkmark 12$ Muzio M, Ni J, Feng P, Dixit VM: IRAK (Pelle) family member IRAK-2 and MyD88 as proximal mediators of IL-1 signaling. Science 1997;278:1612-1615. 
13 Suzuki N, Suzuki S, Millar DG, Unno M, Hara $\mathrm{H}$, Calzascia T, et al: A critical role for the innate immune signaling molecule IRAK- 4 in T cell activation. Science 2006;311:1927-1932.

14 Li Q, Verma IM: NF-kappaB regulation in the immune system. Nat Rev Immunol 2002;2: 725-734.

-15 Franchi L, Warner N, Viani K, Nuñez G: Function of NOD-like receptors in microbial recognition and host defense. Immunol Rev 2009;227:106-128.

- 16 Shigeoka AA, Kambo A, Mathison JC, King AJ, Hall WF, da Silva Correia J, et al: NOD1 and NOD2 are expressed in human and murine renal tubular epithelial cells and participate in renal ischemia reperfusion injury. J Immunol 2010;184:2297-2304.

17 Schroder K, Tschopp J: The inflammasomes. Cell 2010;140:821-832.

18 Onomoto K, Yoneyama M, Fujita T: Regulation of antiviral innate immune responses by RIG-I family of RNA helicases. Curr Top Microbiol Immunol 2007;316:193-205.

$\checkmark 19$ De Nardo D: Toll-like receptors: activation, signalling and transcriptional modulation. Cytokine 2015;74:181-189.

20 Leemans JC, Kors L, Anders HJ, Florquin S: Pattern recognition receptors and the inflammasome in kidney disease. Nat Rev Nephrol 2014;10:398-414.

-21 Salvadori M, Rosso G, Bertoni E: Complement involvement in kidney diseases: from physiopathology to therapeutical targeting. World J Nephrol 2015;4:169-184.

22 Daha MR: Role of complement in innate immunity and infections. Crit Rev Immunol 2010;30:47-52.

-23 De Vries B, Walter SJ, Peutz-Kootstra CJ, Wolfs TG, van Heurn LW, Buurman WA: The mannose-binding lectin-pathway is involved in complement activation in the course of renal ischemia-reperfusion injury. Am J Pathol 2004;165:1677-1688.

24 Lech M, Rommele C, Anders HJ: Pentraxins in nephrology: C-reactive protein, serum amyloid $P$ and pentraxin-3. Nephrol Dial Transplant 2013;28:803-811.

-25 Bottazzi B, Garlanda C, Salvatori G, Jeannin P, Manfredi A, Mantovani A: Pentraxins as a key component of innate immunity. Curr Opin Immunol 2006;18:10-15.

-26 Nauta AJ, de Haij S, Bottazzi B, Mantovani A, Borrias MC, Aten J, et al: Human renal epithelial cells produce the long pentraxin PTX3 Kidney Int 2005;67:543-553.

27 Lech M, Römmele C, Gröbmayr R, Eka Susanti H, Kulkarni OP, Wang S, et al: Endogenous and exogenous pentraxin-3 limits postischemic acute and chronic kidney injury. Kidney Int 2013;83:647-661.

28 Oliveira G, Xavier P, Sampaio S, Alves H: Thymoglobulin down-regulates pentraxin 3 synthesis that correlates with acute rejection on kidney transplant patients. Tissue Antigens 2010;75:594-595.

29 Land WG: Emerging role of innate immunity in organ transplantation part II: potential of damage-associated molecular patterns to generate immunostimulatory dendritic cells. Transplant Rev (Orlando) 2012;26:73-87.

30 Bianchi ME: HMGB1 loves company. J Leukoc Biol 2009;86:573-576.

31 Bethke K, Staib F, Distler M, Schmitt U, Jonuleit $\mathrm{H}$, Enk $\mathrm{AH}$, et al: Different efficiency of heat shock proteins (HSP) to activate human monocytes and dendritic cells: superiority of HSP60. J Immunol 2002;169:6141-6148.

32 Land WG: Role of heat shock protein 70 in innate alloimmunity. Front Immunol 2011;2: 89.

33 Shrivastava S, McVey JH, Dorling A: The interface between coagulation and immunity. Am J Transplant 2007;7:499-506.

- 34 Westendorp WH, Leuvenink HG, Ploeg RJ: Brain death induced renal injury. Curr Opin Organ Transplant 2011;16:151-156.

35 Van Werkhoven MB, Damman J, van Dijk MC, Daha MR, de Jong IJ, Leliveld A, et al: Complement mediated renal inflammation induced by donor brain death: role of renal C5a-C5aR interaction. Am J Transplant 2013; 13:875-882

-36 El-Sawy T, Miura M, Fairchild R: Early T cell response to allografts occurring prior to alloantigen priming up-regulates innate-mediated inflammation and graft necrosis. Am J Pathol 2004;165:147-157.

37 Linkermann A, Green DR: Necroptosis. N Engl J Med 2014;370:455-465.

- 38 Dixon SJ, Lemberg KM, Lamprecht MR, Skouta R, Zaitsev EM, Gleason CE, et al: Ferroptosis: an iron-dependent form of nonapoptotic cell death. Cell 2012;149:10601072.

39 Sallustio F, Costantino V, Cox SN, Loverre A, Divella C, Rizzi M, et al: Human renal stem/ progenitor cells repair tubular epithelial cell injury through TLR2-driven inhibin-A and microvesicle-shuttled decorin. Kidney Int 2013;83:392-403.

40 Kulkarni OP, Hartter I, Mulay SR, Hagemann J, Darisipudi MN, Kumar VR S, et al: Toll-like receptor 4-induced IL-22 accelerates kidney regeneration. J Am Soc Nephrol 2014;25:978989.

41 Hanidziar D, Koulmanda M: Inflammation and the balance of Treg and Th17 cells in transplant rejection and tolerance. Curr Opin Organ Transplant 2010;15:411-415.

42 Anders HJ, Schaefer L: Beyond tissue injurydamage-associated molecular patterns, tolllike receptors, and inflammasomes also drive regeneration and fibrosis. J Am Soc Nephrol 2014;25:1387-1400.

43 Lee S, Huen S, Nishio H, Nishio S, Lee HK, Choi BS, et al: Distinct macrophage phenotypes contribute to kidney injury and repair. J Am Soc Nephrol 2011;22:317-326.

44 Zhang MZ, Yao B, Yang S, Jiang L, Wang S, Fan X, et al: CSF-1 signaling mediates recovery from acute kidney injury. J Clin Invest 2012;122:4519-4532.

45 Menke J, Iwata Y, Rabacal WA, Basu R, Yeung YG, Humphreys BD, et al: CSF-1 signals di- rectly to renal tubular epithelial cells to mediate repair in mice. J Clin Invest 2009;119: 2330-2342.

-46 Lech M, Gröbmayr R, Ryu M, Lorenz G, Hartter I, Mulay SR, et al: Macrophage phenotype controls long-term AKI outcomes - kidney regeneration versus atrophy. J Am Soc Nephrol 2014;25:292-304.

47 Chong AS, Alegre ML: Transplantation tolerance and its outcome during infections and inflammation. Immunol Rev 2014;258:80101.

48 Chong AS, Alegre ML: The impact of infection and tissue damage in solid-organ transplantation. Nat Rev Immunol 2012;12:459471

-49 Jang HR, Gandolfo MT, Ko GJ, Satpute S, Racusen L, Rabb H: Early exposure to germs modifies kidney damage and inflammation after experimental ischemia-reperfusion injury. Am J Physiol Renal Physiol 2009;297: F1457-F1465.

50 Lee JR, Muthukumar T, Dadhania D, Toussaint NC, Ling L, Pamer E, et al: Gut microbial community structure and complications after kidney transplantation: a pilot study. Transplantation 2014;98:697-705.

51 Bartman C, Chong AS, Alegre ML: The influence of the microbiota on the immune response to transplantation. Curr Opin Organ Transplant 2015;20:1-7.

-52 Lee JR, Muthukumar T, Dadhania D, Taur Y, Jenq RR, Toussaint NC, et al: Gut microbiota and tacrolimus dosing in kidney transplantation. PLoS One 2015;10:e0122399.

-53 Divito SJ, Wang Z, Shufesky WJ, Liu Q, Tkacheva OA, Montecalvo A, et al: Endogenous dendritic cells mediate the effects of intravenously injected therapeutic immunosuppressive dendritic cells in transplantation. Blood 2010;116:2694-2705.

54 Wood KJ, Zaitsu M, Goto R: Cell mediated rejection. Methods Mol Biol 2013;1034:7183.

55 Stauber DJ, Debler EW, Horton PA, Smith KA, Wilson IA: Crystal structure of the IL-2 signaling complex: paradigm for a heterotrimeric cytokine receptor. Proc Natl Acad Sci U S A 2006;103:2788-2793.

56 Brown EJ, Albers MW, Shin TB, Ichikawa K, Keith CT, Lane WS, et al: A mammalian protein targeted by G1-arresting rapamycin-receptor complex. Nature 1994;369: 756-758.

57 Trinchieri G: Interleukin-12 and the regulation of innate resistance and adaptive immunity. Nat Rev Immunol 2003;3:133-146.

58 Harrington LE, Hatton RD, Mangan PR, Turner H, Murphy TL, Murphy KM, et al: Interleukin 17-producing CD4+ effector T cells develop via a lineage distinct from the $T$ helper type 1 and 2 lineages. Nat Immunol 2005; 6:1123-1132

59 Zhang B, Ramesh G, Uematsu S, Akira S, Reeves WB: TLR4 signaling mediates inflammation and tissue injury in nephrotoxicity. J Am Soc Nephrol 2008;19:923-932. 
60 Leemans JC, Stokman G, Claessen N, Rouschop KM, Teske GJ, Kirschning CJ, et al: Renal-associated TLR2 mediates ischemia/reperfusion injury in the kidney. J Clin Invest 2005;115:2894-2903.

$61 \mathrm{Wu} \mathrm{H}$, Noordmans GA, O’Brien MR, Ma J, Zhao CY, Zhang GY, et al: Absence of MyD88 signaling induces donor-specific kidney allograft tolerance. J Am Soc Nephrol 2012;23: 1701-1716.

62 Tesar BM, Zhang J, Li Q, Goldstein DR: TH1 immune responses to fully MHC mismatched allografts are diminished in the absence of MyD88, a toll-like receptor signal adaptor protein. Am J Transplant 2004;4:1429-1439.

-63 Krüger B, Krick S, Dhillon N, Lerner SM, Ames S, Bromberg JS, et al: Donor toll-like receptor 4 contributes to ischemia and reperfusion injury following human kidney transplantation. Proc Natl Acad Sci U S A 2009; 106:3390-3395.

64 Reilly M, Miller RM, Thomson MH, Patris V, Ryle P, McLoughlin L, et al: Randomized, double-blind, placebo-controlled, dose-esca- lating phase I, healthy subjects study of intravenous OPN-305, a humanized anti-TLR2 antibody. Clin Pharmacol Ther 2013;94:593600.

65 Lassen S, Lech M, Römmele C, Mittruecker HW, Mak TW, Anders HJ: Ischemia reperfusion induces IFN regulatory factor 4 in renal dendritic cells, which suppresses postischemic inflammation and prevents acute renal failure. J Immunol 2010;185:1976-1983.

66 Noris M, Cassis P, Azzollini N, Cavinato R, Cugini D, Casiraghi F, et al: The Toll-IL-1R member Tir8/SIGIRR negatively regulates adaptive immunity against kidney grafts. J Immunol 2009; 183:4249-4260.

67 Yang H, Hreggvidsdottir HS, Palmblad K, Wang H, Ochani M, Li J, et al: A critical cysteine is required for HMGB1 binding to tolllike receptor 4 and activation of macrophage cytokine release. Proc Natl Acad Sci U S A 2010;107:11942-11947.

68 Li J, Gong Q, Zhong S, Wang L, Guo H, Xiang $Y$, et al: Neutralization of the extracellular HMGB1 released by ischaemic damaged renal cells protects against renal ischaemia-reperfusion injury. Nephrol Dial Transplant 2011; 26:469-478.

69 Musumeci D, Roviello GN, Montesarchio D: An overview on HMGB1 inhibitors as potential therapeutic agents in HMGB1-related pathologies. Pharmacol Ther 2014;141:347357.

70 Chandraker A, Azuma H, Nadeau K, Carpenter CB, Tilney NL, Hancock WW, et al: Late blockade of $\mathrm{T}$ cell costimulation interrupts progression of experimental chronic allograft rejection. J Clin Invest 1998;101:2309-2318.

71 Gilmore TD, Herscovitch M: Inhibitors of NF-kappaB signaling: 785 and counting. Oncogene 2006;25:6887-6899.

72 Quinn EM, Wang JH, O'Callaghan G, Redmond HP: MicroRNA-146a is upregulated by and negatively regulates TLR2 signaling. PLoS One 2013;8:e62232.

-73 Oberbarnscheidt MH, Zeng Q, Li Q, Dai H, Williams AL, Shlomchik WD, et al: Non-self recognition by monocytes initiates allograft rejection. J Clin Invest 2014;124:3579-3589. 